\title{
Independent Process Analysis without A Priori Dimensional Information
}

\author{
Barnabás Póczos, Zoltán Szabó, Melinda Kiszlinger, and András Lőrincz* \\ Department of Information Systems \\ Eötvös Loránd University, Budapest, Hungary \\ \{pbarn,szzoli\}@cs.elte.hu, \{kmelinda, andras.lorincz\}@elte.hu
}

\begin{abstract}
Recently, several algorithms have been proposed for independent subspace analysis where hidden variables are i.i.d. processes. We show that these methods can be extended to certain AR, MA, ARMA and ARIMA tasks. Central to our paper is that we introduce a cascade of algorithms, which aims to solve these tasks without previous knowledge about the number and the dimensions of the hidden processes. Our claim is supported by numerical simulations. As a particular application, we search for subspaces of facial components.
\end{abstract}

\section{Introduction}

Independent Subspace Analysis (ISA), also known as Multidimensional Independent Component Analysis [1, is a generalization of Independent Component Analysis (ICA). ISA assumes that certain sources depend on each other, but the dependent groups of sources are still independent of each other, i.e., the independent groups are multidimensional. The ISA task has been subject of extensive research [1/2]3/4/5/6/789. In this case, one assumes that the hidden sources are independent and identically distributed (i.i.d.) in time. Temporal independence is, however, a gross oversimplification of real sources including acoustic or biomedical data. One may try to overcome this problem, by assuming that hidden processes are, e.g., autoregressive (AR) processes. Then we arrive to the AR Independent Process Analysis (AR-IPA) task [10]11]. Another method to weaken the i.i.d. assumption is to assume moving averaging (MA). This direction is called Blind Source Deconvolution (BSD) [12, in this case the observation is a temporal mixture of the i.i.d. components.

The AR and MA models can be generalized and one may assume ARMA sources instead of i.i.d. ones. As an additional step, the method can be extended to non-stationary integrated ARMA (ARIMA) processes, which are important, e.g., for modelling economic processes [13].

In this paper, we formulate the AR-, MA-, ARMA-, ARIMA-IPA generalization of the ISA tasks, when (i) one allows for multidimensional hidden components and (ii) the dimensions of the hidden processes are not known. We show that in the undercomplete case, when the number of 'sensors' is larger than the number of 'sources', these tasks can be reduced to the ISA task.

\footnotetext{
* Corresponding author
} 


\section{Independent Subspace Analysis}

The ISA task can be formalized as follows:

$$
\mathbf{x}(t)=\mathbf{A e}(t), \text { where } \mathbf{e}(t)=\left[\mathbf{e}^{1}(t) ; \ldots ; \mathbf{e}^{M}(t)\right] \in \mathbb{R}^{D_{e}}
$$

and $\mathbf{e}(t)$ is a vector concatenated of components $\mathbf{e}^{m}(t) \in \mathbb{R}_{e}^{d_{e}^{m}}$. The total dimension of the components is $D_{e}=\sum_{m=1}^{M} d_{e}^{m}$. We assume that for a given $m, \mathbf{e}^{m}(t)$ is i.i.d. in time $t$, and sources $\mathbf{e}^{m}$ jointly independent, i.e., $I\left(\mathbf{e}^{1}, \ldots, \mathbf{e}^{M}\right)=0$, where $I($.$) denotes the mutual information (MI) of the arguments. The dimen-$ sion of observation $\mathbf{x}$ is $D_{x}$. Assume that $D_{x}>D_{e}$, and $\mathbf{A} \in \mathbb{R}^{D_{x} \times D_{e}}$ is of full column rank. Under these conditions, one may assume without any loss of generality that both the observed $(\mathbf{x})$ and the hidden $(\mathbf{e})$ signals are white. For example, one may apply Principal Component Analysis (PCA) as a preprocessing stage. Then the ambiguities of the ISA task are as follows [14]: Sources can be determined up to permutation and up to orthogonal transformations within the subspaces.

\subsection{The ISA Separation Theorem}

We are to uncover the independent subspaces. Our task is to find a matrix $\mathbf{W} \in \mathbb{R}^{D_{e} \times D_{x}}$ such that $\mathbf{y}(t)=\mathbf{W} \mathbf{x}(t), \mathbf{y}(t)=\left[\mathbf{y}^{1}(t) ; \ldots ; \mathbf{y}^{M}(t)\right], \mathbf{y}^{m}=$ $\left[y_{1}^{m} ; \ldots ; y_{d_{e}^{m}}^{m}\right] \in \mathbb{R}^{d_{e}^{m}},(m=1, \ldots, M)$ with the condition that components $\mathbf{y}^{m}$ are independent. Here, (i) $y_{i}^{m}$ denotes the $i^{\text {th }}$ coordinate of the $m^{\text {th }}$ estimated subspace, and (ii) $\mathbf{W}$ can be chosen to be orthogonal because of the whitening assumption. This task can be solved by means of cost function that aims to minimize the mutual information between components:

$$
J_{1}(\mathbf{W}) \doteq I\left(\mathbf{y}^{1}, \ldots, \mathbf{y}^{M}\right) .
$$

One can rewrite $J_{1}(\mathbf{W})$ as follows:

$$
J_{2}(\mathbf{W}) \doteq I\left(y_{1}^{1}, \ldots, y_{d_{e}^{M}}^{M}\right)-\sum_{m=1}^{M} I\left(y_{1}^{m}, \ldots, y_{d_{e}^{m}}^{m}\right) .
$$

The first term of the r.h.s. is the ICA cost function; it aims to minimize mutual information for all coordinates. The other term is a kind of anti-ICA term; it aims to maximize mutual information within the subspaces. One may try to apply a heuristics and to optimize (3) in order: (1) Start by any 'infomax' ICA algorithm and minimize the first term of the r.h.s. in (3). (2) Apply only permutations to the coordinates such that they optimize the second term. In this second step coordinates are not changed, but (3) may decrease further. Surprisingly, this heuristics leads to the global minimum of $(2)$ in many cases. In other words, in many cases, ICA that minimizes the first term of the r.h.s. of (3) solves the ISA task apart from the grouping of the coordinates into subspaces. This feature was observed by Cardoso, first [1]. The extent of this feature is still an open issue. 
Nonetheless, we call it 'Separation Theorem', because for elliptically symmetric sources and for some other distribution types one can prove that it is rigorously true [15]. (See also, the result concerning local minimum points [16]). Although there is no proof for general sources as of yet, a number of algorithms applies this heuristics with success [14]16]17|18]19.

\subsection{ISA with Unknown Components}

Another issue concerns the computation of the second term of (3). If the $d_{e}^{m}$ dimensions of subspaces $\mathbf{e}^{m}$ are known then one might rely on multi-dimensional entropy estimations [9], but these are computationally expensive. Other methods deal with implicit or explicit pair-wise dependency estimations [17]16. Interestingly, if the observations are indeed from an ICA generative model, then the minimization of the pair-wise dependencies is sufficient to get the solution of the ICA task according to the Darmois-Skitovich theorem [20]. This is not the case for the ISA task, however. There are ISA tasks, where the estimation of pair-wise dependencies is insufficient for recovering the hidden subspaces [9. Nonetheless, such algorithms seem to work nicely in many practical cases.

A further complication arises if the $d_{e}^{m}$ dimensions of subspaces $\mathbf{e}^{m}$ are not known. Then the dimension of the entropy estimation becomes uncertain. Methods that try to apply pair-wise dependencies were proposed to this task. One can find a block-diagonalization method in [16, whereas [17] makes use of kernel estimations of the mutual information.

Here we shall assume that the separation theorem is satisfied. We shall apply ICA preprocessing. This step will be followed by the estimation of the pair-wise mutual information of the ICA coordinates. These quantities will be considered as the weights of a weighted graph, the vertices of the graph being the ICA coordinates. We shall search for clusters of this graph. In our numerical studies, we make use of Kernel Canonical Correlation Analysis [5] for the MI estimation. A variant of the Ncut algorithm [21] is applied for clustering. As a result, the mutual information within (between) cluster(s) becomes large (small).

The problem is that this ISA method requires i.i.d. hidden sources. Below, we show how to generalize the ISA task to more realistic sources. Finally, we solve this more general problem when the dimension of the subspaces is not known.

\section{ISA Generalizations}

We need the following notations: Let $z$ stand for the time-shift operation, that is $(z \mathbf{v})(t):=\mathbf{v}(t-1)$. The $\mathrm{N}$ order polynomials of $D_{1} \times D_{2}$ matrices are denoted as $\left.\mathbb{R}[z]_{N}^{D_{1} \times D_{2}}:=\left\{\mathbf{F}[z]=\sum_{n=0}^{N} \mathbf{F}_{n} z^{n}, \mathbf{F}_{n} \in \mathbb{R}^{D_{1} \times D_{2}}\right)\right\}$. Let $\nabla^{r}[z]:=(\mathbf{I}-\mathbf{I} z)^{r}$ denote the operator of the $r^{t h}$ order difference, where $\mathbf{I}$ is the identity matrix, $r \geq 0, r \in \mathbb{Z}$.

Now, we are to estimate unknown components $\mathbf{e}^{m}$ from observed signals $\mathbf{x}$. We always assume that $\mathbf{e}$ takes the form like in (1) and that $\mathbf{A} \in \mathbb{R}^{D_{x} \times D_{s}}$ is of full column rank. 
1. AR-IPA: The AR generalization of the ISA task is defined by the following equations: $\mathbf{x}=\mathbf{A s}$, where $\mathbf{s}$ is an $\mathrm{AR}(\mathrm{p})$ process i.e, $\mathbf{P}[z] \mathbf{s}=\mathbf{Q e}$, $\mathbf{Q} \in \mathbb{R}^{D_{s} \times D_{e}}$, and $\mathbf{P}[z]:=\mathbf{I}_{D_{s}}-\sum_{i=1}^{p} \mathbf{P}_{i} z^{i} \in \mathbb{R}[z]_{p}^{D_{s} \times D_{s}}$. We assume that $\mathbf{P}[z]$ is stable, that is $\operatorname{det}(\mathbf{P}[z] \neq 0)$, for all $z \in \mathbb{C},|z| \leq 1$. For $d_{e}^{m}=1$ this task was investigated in [10]. Case $d_{e}^{m}>1$ is treated in [11]. The special case of $p=0$ is the ISA task.

2. MA-IPA or Blind Subspace Deconvolution (BSSD) task: The ISA task is generalized to blind deconvolution task (moving average task, MA(q)) as follows: $\mathbf{x}=\mathbf{Q}[z] \mathbf{e}$, where $\mathbf{Q}[z]=\sum_{j=0}^{q} \mathbf{Q}_{j} z^{j} \in \mathbb{R}[z]_{q}^{D_{x} \times D_{e}}$.

3. ARMA-IPA task: The two tasks above can be merged into a model, where the hidden $\mathbf{s}$ is $\operatorname{ARMA}(\mathrm{p}, \mathrm{q}): \mathbf{x}=\mathbf{A s}, \mathbf{P}[z] \mathbf{s}=\mathbf{Q}[z] \mathbf{e}$. Here $\mathbf{P}[z] \in \mathbb{R}[z]_{p}^{D_{s} \times D_{s}}$, $\mathbf{Q}[z] \in \mathbb{R}[z]_{q}^{D_{s} \times D_{e}}$. We assumed that $\mathbf{P}[z]$ is stable. Thus the ARMA process is stationary.

4. ARIMA-IPA task: In practice, hidden processes $\mathbf{s}$ may be non-stationary. ARMA processes can be generalized to the non-stationary case. This generalization is called integrated ARMA, or $\operatorname{ARIMA}(p, r, q)$. The assumption here is that the $r^{t h}$ difference of the process is an ARMA process. The corresponding IPA task is then

$$
\mathbf{x}=\mathbf{A s}, \text { where } \mathbf{P}[z] \nabla^{r}[z] \mathbf{s}=\mathbf{Q}[z] \mathbf{e} .
$$

\section{Reduction of ARIMA-IPA to ISA}

We show how to solve the above tasks by means of ISA algorithms. We treat the ARIMA task. Others are special cases of this one. In what follows, we assume that: (i) $\mathbf{P}[z]$ is stable, (ii) the mixing matrix $\mathbf{A}$ is of full column rank, and (iii) $\mathbf{Q}[z]$ has left inverse. In other words, there exists a polynomial matrix $\mathbf{W}[z] \in \mathbb{R}[z]^{D_{e} \times D_{s}}$ such that $\mathbf{W}[z] \mathbf{Q}[z]=\mathbf{I}_{D_{e}} 1$

The route of the solution is elaborated here. Let us note that differentiating the observation $\mathbf{x}$ of the ARIMA-IPA task in Eq. (4) in $r^{\text {th }}$ order, and making use of the relation $z \mathbf{x}=\mathbf{A}(z \mathbf{s})$, the following holds:

$$
\nabla^{r}[z] \mathbf{x}=\mathbf{A}\left(\nabla^{r}[z] \mathbf{s}\right) \text {, and } \mathbf{P}[z]\left(\nabla^{r}[z] \mathbf{s}\right)=\mathbf{Q}[z] \mathbf{e} .
$$

That is taking $\nabla^{r}[z] \mathbf{x}$ as observations, one ends up with an ARMA-IPA task. Assume that $D_{x}>D_{e}$ (undercomplete case). We call this task uARMA-IPA. Now we show how to transform the uARMA-IPA task to ISA. The method is similar to that of [23] where it was applied for BSD.

Theorem If the above assumptions are fulfilled then in the uARMA-IPA task, observation process $\mathbf{x}(t)$ is autoregressive and its innovation $\tilde{\mathbf{x}}(t):=\mathbf{x}(t)-$ $E[\mathbf{x}(t) \mid \mathbf{x}(t-1), \mathbf{x}(t-2), \ldots]=\mathbf{A Q}_{0} \mathbf{e}(t)$, where $E[\cdot \cdot \cdot]$ denotes the conditional expectation value. Consequently, there is a polynomial matrix $\mathbf{W}_{\mathrm{AR}}[z] \in \mathbb{R}[z]^{D_{x} \times D_{x}}$ such that $\mathbf{W}_{\mathrm{AR}}[z] \mathbf{x}=\mathbf{A} \mathbf{Q}_{0} \mathbf{e}$.

\footnotetext{
${ }^{1}$ One can show for $D_{s}>D_{e}$ that under mild conditions $\mathbf{Q}[z]$-has an inverse with probability $1\left[22\right.$; e.g., when the matrix $\left[\mathbf{Q}_{0}, \ldots, \mathbf{Q}_{q}\right]$ is drawn from a continuous distribution.
} 
Proof Steps of the proof:

1. In the uARMA-IPA task the following equations hold:

$$
\begin{aligned}
\mathbf{P}[z] \mathbf{s} & =\mathbf{Q}[z] \mathbf{e} \\
\mathbf{x} & =\mathbf{A} \mathbf{s}
\end{aligned}
$$

or equivalently

$$
\begin{aligned}
& \mathbf{s}(t)=\sum_{i=1}^{p} \mathbf{P}_{i} \mathbf{s}(t-i)+\sum_{j=0}^{q} \mathbf{Q}_{j} \mathbf{e}(t-j), \\
& \mathbf{x}(t)=\mathbf{A} \mathbf{s}(t)
\end{aligned}
$$

Non-degenerate linear transformation of an ARMA process is also ARMA. Thus, observation process $\mathbf{x}$ is an ARMA process. Formally: Substituting $\mathbf{s}(t)$ of Eq. (8) into Eq. (9) and then using the pseudoinverse of matrix $\mathbf{A}$ and expression $\mathbf{s}(t)=\mathbf{A}^{-1} \mathbf{x}(t)$ that follows from $E q$. (9), we have

$$
\mathbf{x}(t)=\sum_{i=1}^{p} \mathbf{A} \mathbf{P}_{i} \mathbf{A}^{-1} \mathbf{x}(t-i)+\sum_{j=0}^{q} \mathbf{A Q}_{j} \mathbf{e}(t-j) .
$$

Process $\mathbf{e}(t)$ is i.i.d, so the process $\mathbf{x}(t)$ is $A R M A$.

2. We assumed that $\mathbf{Q}[z]$ has left inverse and thus $\mathbf{e}$ of $E q$. (6) can be expressed from $\mathbf{s}$ via multiplication with a polynomial matrix. One says that $\mathbf{e}$ derives from $\mathbf{s}$ by causal FIR filtering. The same holds for $\mathbf{x}$ because of Eq. (9):

$$
\mathbf{e}=\mathbf{P}^{\prime}[z] \mathbf{s}=\mathbf{P}^{\prime}[z]\left(\mathbf{A}^{-1} \mathbf{x}\right)=\left(\mathbf{P}^{\prime}[z] \mathbf{A}^{-1}\right) \mathbf{x}=: \mathbf{P}^{\prime \prime}[z] \mathbf{x},
$$

where $\mathbf{P}^{\prime}[z]:=\mathbf{Q}^{-1}[z] \mathbf{P}[z]=\sum_{n=0}^{N} \mathbf{P}_{n}^{\prime} z^{-n} \in \mathbb{R}[z]_{N}^{D_{e} \times D_{s}}, \mathbf{P}^{\prime \prime}[z] \in \mathbb{R}[z]_{N}^{D_{e} \times D_{x}}$ and $N$ denotes the degree of the polynomials.

3. The first term of the r.h.s. of the observation $\mathbf{x}$ in Eq. (10) is a linear expression of a finite history of $\mathbf{x}$. Equation (11) implies, that the second term, except $\mathbf{A Q}_{0} \mathbf{e}(t)$, also belongs to the linear hull of the finite history of $\mathbf{x}$. Formally:

$$
\begin{aligned}
\mathbf{x}(t) & =\mathbf{A} \mathbf{Q}_{0} \mathbf{e}(t)+\sum_{i=1}^{p} \mathbf{A} \mathbf{P}_{i} \mathbf{A}^{-1} \mathbf{x}(t-i)+\sum_{j=1}^{q} \mathbf{A Q}_{j}\left(\mathbf{P}^{\prime \prime}[z] \mathbf{x}\right)(t-j) \\
& \in \mathbf{A} \mathbf{Q}_{0} \mathbf{e}(t)+\langle\mathbf{x}(t-1), \mathbf{x}(t-2), \ldots, \mathbf{x}(t-\max (p, q+N))\rangle
\end{aligned}
$$

4. $\mathbf{e}(t)$ is independent of $\langle\mathbf{x}(t-1), \mathbf{x}(t-2), \ldots, \mathbf{x}(t-\max (p, q+N))\rangle$. Consequently, observation process $\mathbf{x}(t)$ is autoregressive with innovation $\mathbf{A Q}_{0} \mathbf{e}(t)$.

Thus, AR fit of $\mathbf{x}(t)$ can be used for the estimation of $\mathbf{A Q}_{0} \mathbf{e}(t)$. This innovation corresponds to the observation of an undercomplete ISA model $\left(D_{x}>D_{e}\right)^{2}$,

${ }^{2}$ Assumptions made for $\mathbf{Q}[z]$ and $\mathbf{A}$ in the uARMA-IPA task implies that $\mathbf{A Q}_{0}$ is of full column rank and thus the resulting ISA task is well defined. 
which can be reduced to a complete ISA $\left(D_{x}=D_{e}\right)$ using PCA. Finally, the solution can be finished by any ISA procedure. The reduction procedure implies that hidden components $\mathbf{e}^{m}$ can be recovered only up to the ambiguities of the ISA task: components of (identical dimensions) can be recovered only up to permutations. Within each subspaces, unambiguity is warranted only up to orthogonal transformations.

The steps of our algorithm are summarized in Table 1.

Table 1: Pseudocode of the undercomplete ARIMA-IPA algorithm

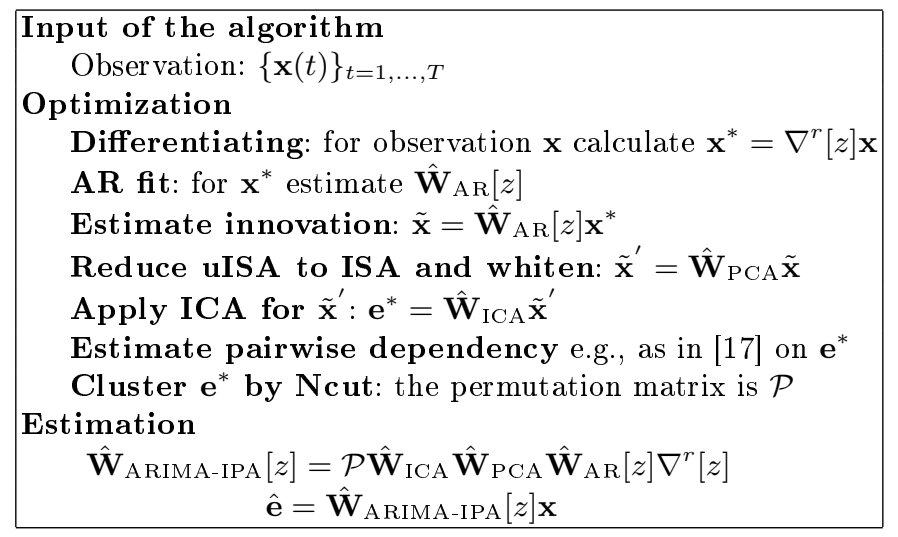

\section{$5 \quad$ Results}

In this section we demonstrate the theoretical results by numerical simulations.

\subsection{ARIMA Processes}

We created a database for the demonstration: Hidden sources $\mathbf{e}^{m}$ are 4 pieces of $2 \mathrm{D}, 3$ pieces of $3 \mathrm{D}, 2$ pieces of $4 \mathrm{D}$ and 1 piece of $5 \mathrm{D}$ stochastic variables, i.e., $M=10$. These stochastic variables are independent, but the coordinates of each stochastic variable $\mathbf{e}^{m}$ depend on each other. They form a 30 dimensional space together $\left(D_{e}=30\right)$. For the sake of illustration, 3D (2D) sources emit random samples of uniform distributions defined on different 3D geometrical forms (letters of the alphabet). The distributions are depicted in Fig. 1a (Fig. 1b). 30,000 samples were drawn from the sources and they were used to drive an $\operatorname{ARIMA}(2,1,6)$ process defined by (4). Matrix $\mathbf{A} \in \mathbb{R}^{60 \times 60}$ was randomly generated and orthogonal. We also generated polynomial $\mathbf{Q}[z] \in \mathbb{R}[z]_{5}^{60 \times 30}$ and stable polynomial $\mathbf{P}[z] \in \mathbb{R}[z]_{1}^{60 \times 60}$ randomly. The visualization of the 60 dimensional process is hard to illustrate: a typical 3D projection is shown in Fig. 1c. The task is to estimate original sources $\mathbf{e}^{m}$ using these non-stationary observations. 
$r^{\text {th }}$-order differencing of the observed ARIMA process gives rise to an ARMA process. Typical 3D projection of this ARMA process is shown Fig. 1d Now, one can execute the other steps of Table 1 and these steps provide the estimations of the hidden components $\hat{\mathbf{e}}^{m}$. Estimations of the 3D (2D) components are provided in Fig. 1e (Fig. 1f). In the ideal case, the product of matrix $\mathbf{A Q}_{0}$ and the matrices provided by PCA and ISA, i.e., $\mathbf{G}:=\left(\mathcal{P} \hat{\mathbf{W}}_{\mathrm{ICA}} \hat{\mathbf{W}}_{\mathrm{PCA}}\right) \mathbf{A Q}_{0} \in \mathbb{R}^{D_{e} \times D_{e}}$ is a block permutation matrix made of $d_{e}^{m} \times d_{e}^{m}$ blocks. This is shown in Fig. 1g.

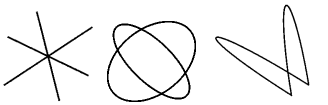

(a)

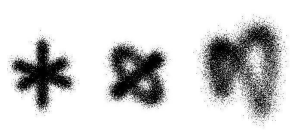

(e)

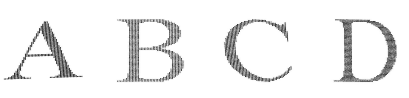

(b)

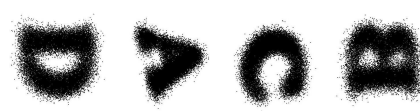

(f)

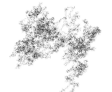

(c)

Fig. 1: (a-b) components of the database. (a): 3 pieces of 3D geometrical forms, (b): 4 pieces of $2 \mathrm{D}$ letters. Hidden sources are uniformly distributed variables on these objects. (c): typical 3D projection of the observation. (d): typical 3D projection of the $r^{\text {th }}$-order difference of the observation, (e): estimated 2D components, (f): estimated 3D components, $(\mathrm{g})$ : Hinton diagram of $\mathbf{G}$, which - in case of perfect estimation - becomes a block permutation matrix.

\subsection{Facial Components}

We have generated another database using the FaceGen ${ }^{3}$ animation software. In our database we had 800 different front view faces with the 6 basic facial expressions. We had thus 4,800 images in total. All images were sized to $40 \times 40$ pixel. Figure $2 \mathrm{a}$ shows samples of the database. A large $\mathbf{X} \in \mathbb{R}^{4800 \times 1600}$ matrix was compiled; rows of this matrix were 1600 dimensional vectors formed by the pixel values of the individual images. The columns of this matrix were considered as mixed signals. This treatment replicates the experiments in [24]: Bartlett et al., have shown that in such cases, undercomplete ICA finds components resembling to what humans consider facial components. We were interested in seeing the components grouped by undercomplete ISA algorithm. The observed 4800 dimensional signals were compressed by PCA to 60 dimensions and we searched for 4 pieces of ISA subspaces using the algorithm detailed in Table 1 . The 4 subspaces that our algorithm found are shown in Fig. 2b. As it can be

\footnotetext{
3 http://www . facegen.com/modeller.htm
} 
seen, the 4 subspaces embrace facial components which correspond mostly to mouth, eye brushes, facial profiles, and eyes, respectively.

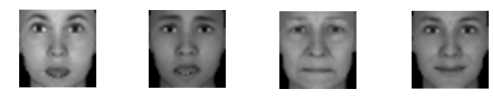

(a)

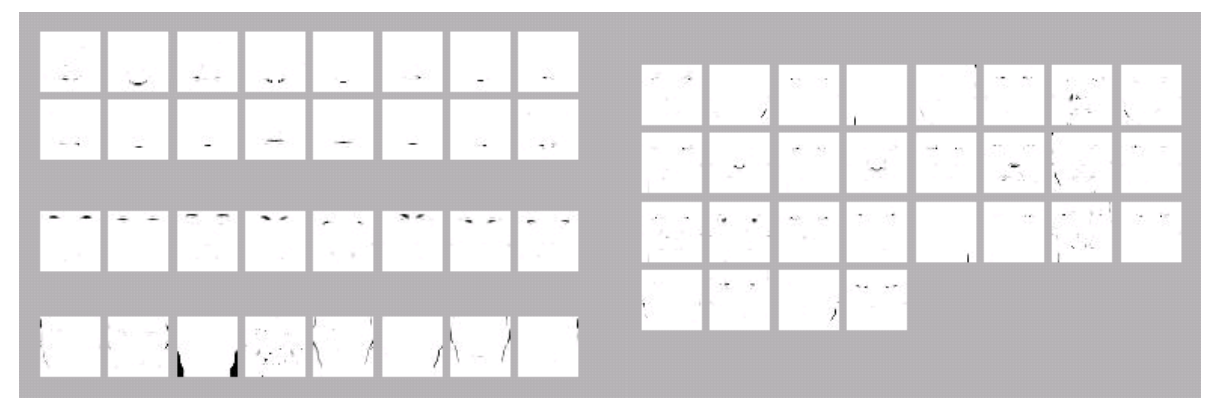

(b)

Fig. 2: (a) Samples from the database. (b) Four subspaces of the components. Components in distinct groups correspond mostly to mouth, eye brushes, facial profiles, and eyes respectively.

\section{Conclusions}

We have extended the ISA task to problems where the hidden components can be AR, MA, ARMA, or ARIMA processes. We showed an algorithm that can identify the hidden subspaces under certain conditions. The algorithm does not require previous knowledge about the dimensions of the subspaces. The working of the algorithm was demonstrated on an artificially generated ARIMA process, as well as on a database of facial expressions.

\section{References}

1. J. F. Cardoso. Multidimensional independent component analysis. In Proc. of ICASSP, volume 4, pages 1941-1944, 1998.

2. A. Hyvärinen and P. O. Hoyer. Emergence of phase and shift invariant features by decomposition of natural images into independent feature subspaces. Neural Computation, 12:1705-1720, 2000.

3. R. Vollgraf and K. Obermayer. Multi-dimensional ICA to separate correlated sources. In Proc. of NIPS, volume 14, pages 993-1000. MIT Press, 2001. 
4. H. Stögbauer, A. Kraskov, S. A. Astakhov, and P. Grassberger. Least dependent component analysis based on mutual information. Physical Review E - Statistical, Nonlinear, and Soft Matter Physics, 70, 2004.

5. F. R. Bach and M. I. Jordan. Beyond independent components: Trees and clusters. Journal of Machine Learning Research, 4:1205-1233, 2003.

6. F. J. Theis. Blind signal separation into groups of dependent signals using joint block diagonalization. In Proc. of ISCAS., pages 5878-5881, 2005.

7. A. Hyvärinen and U. Köster. FastISA: A fast fixed-point algorithm for independent subspace analysis. In Proc. of ESANN, Evere, Belgium, 2006.

8. G. Nolte, F. C. Meinecke, A. Ziehe, and K. R. Müller. Identifying interactions in mixed and noisy complex systems. Physical Review E, 73(051913), 2006.

9. B. Póczos and A. Lốrincz. Independent subspace analysis using geodesic spanning trees. In Proc. of ICML, pages 673-680, New York, NY, USA, 2005. ACM Press.

10. A. Hyvärinen. Independent component analysis for time-dependent stochastic processes. In Proc. of ICANN, pages 541-546, Berlin, 1998. Springer-Verlag.

11. B. Póczos, B. Takács, and A. Lôrincz. Independent subspace analysis on innovations. In Proc. of ECML, pages 698-706, Berlin, 2005. Springer-Verlag.

12. S. Choi, A. Cichocki, H.-M. Park, and S.-Y. Lee. Blind source separation and independent component analysis. Neural Information Processing - Letters and Reviews, 6:1-57, 2005.

13. T. C. Mills. Time Series Techniques for Economists. Cambridge University Press, Cambridge, 1990.

14. F. J. Theis. Uniqueness of complex and multidimensional independent component analysis. Signal Processing, 84(5):951-956, 2004.

15. Z. Szabó, B. Póczos, and A. Lőrincz. Separation theorem for $\mathbb{K}$-independent subspace analysis with sufficient conditions. Technical report, Eötvös Loránd University, Budapest, 2006. http://arxiv.org/abs/math.ST/0608100

16. F. J. Theis. Towards a general independent subspace analysis. In Proc. of NIPS, 2007.

17. F. R. Bach and M. I. Jordan. Finding clusters in Independent Component Analysis. In Proc. of ICA2003, pages 891-896, 2003.

18. Z. Szabó, B. Póczos, and A. Lórincz. Cross-entropy optimization for independent process analysis. In Proc. of ICA2006, LNCS 3889, pages 909-916. Springer, 2006.

19. K. Abed-Meraim and A. Belouchrani. Algorithms for joint block diagonalization. In Proc. of EUSIPCO, pages 209-212, 2004.

20. P. Comon. Independent Component Analysis, a new concept? Signal Processing, Elsevier, 36(3):287-314, April 1994. Special issue on Higher-Order Statistics.

21. S. Yu and J. Shi. Multiclass spectral clustering. In Proc. of ICCV, 2003.

22. R. Rajagopal and L. C. Potter. Multivariate MIMO FIR inverses. IEEE Transactions on Image Processing, 12:458 - 465, 2003.

23. A. Gorokhov and P. Loubaton. Blind identification of MIMO-FIR systems: A generalized linear prediction approach. Signal Processing, 73:105-124, 1999.

24. M. Bartlett, J. Movellan, and T. Sejnowski. Face recognition by independent component analysis. IEEE Tran. on neural networks, 13(6):1450-1464, 2002. 